\title{
Assessment of expression of selected Bcl-2 family proteins in lymphoid infiltration in patients with B-cell chronic lymphocytic leukaemia treated with nucleoside analogues
}

\author{
Dorota Lemancewicz ${ }^{1,2}$, Janusz Dzięciol' ${ }^{1}$,Jarosław Piszcz², Agnieszka Lebelt' ${ }^{1}$, \\ Katarzyna Mazgajska-Barczyk², Beata Klim, Janusz Kłoczko \\ ${ }^{1}$ Department of Human Anatomy and ${ }^{2}$ Department of Haematology, Medical University of Bialystok
}

\begin{abstract}
B-cell chronic lymphocytic leukaemia (B-CLL) is characterized by clonal growth and accumulation of mature lymphoid cells due to disturbance in genetically regulated form of cell death called apoptosis. The intrinsic mechanism of apoptosis is controlled by Bcl-2 family proteins. Purine nucleoside analogues induce the apoptosis in cells in a state of quiescence. The aim of the study was to assess expression of selected Bcl-2 family proteins in neoplastic infiltration in bone marrow in patients with B-CLL treated with nucleoside analogues. The study comprised examination of bone marrow obtained routinely by trephine biopsy from 18 patients with B-CLL diagnosed before administration of purine nucleoside analogues treatment and after its completion. Expression of Bcl-2, Bcl-x and Bax proteins was examined. Lymphoid cells in bone marrow were present in all patients before administration of treatment. After treatment in two patients bone marrow was infiltrated in diffuse pattern, whereas other patients presented nodular pattern of infiltration. The difference between stage of infiltration before and after treatment was statistically significant $(\mathrm{p}<0.002)$. High percentage of infiltration cells with positive anti Bcl-2 reaction from $42.0 \%$ in one patient to $85.33 \pm 3.06 \%$ in four patients before treatment was observed. After treatment percentage of infiltration cells with positive anti Bcl-2 antibody reaction was from $33.0 \pm 18.38 \%$ in two patients to $99.0 \%$ in one patient. Positive correlation between stage of infiltration and expression of Bcl-2 protein was confirmed before and after treatment. Such correlations were not observed in case of Bax and Bcl-x. Strong staining of immunohistochemical reaction of cells in lymphoid infiltration with Bcl-2 antibody was confirmed. There was a difference between Bcl-/Bax ratio before and after treatment. Immunohistochemical assessment of expression of Bcl-2 family proteins in cells of lymphoid infiltration in bone marrow of patients with CLL is an important method in detection of minimal residual disease (MRD) after treatment.
\end{abstract}

Key words: B-CLL - Trephine biopsy - Apoptosis - Bcl-2 - Bax - Bcl-x - Nucleoside analogues

\section{Introduction}

B-cell chronic lymphocytic leukaemia (B-CLL) is a disorder characterized by clonal proliferation and accumulation of mature lymphoid cells of low proliferative potential due to disturbance of preset genetically regulated death of cells called apoptosis [1]. Apoptosis may be induced through the extrinsic or intrinsic pathway. Bcl-2 family proteins trigger the intrinsic, i.a. mitochondrial pathway of apoptosis [2]. Some of these proteins are responsible for precipitating changes typ-

Correspondence: D. Lemancewicz, Dept. of Human Anatomy, Medical University of Bialystok, 15-089 Białystok, Mickiewicza Str. 2A, Poland; tel./fax.: (+4885) 7485664,

tel.: (+4885) 7485661, e-mail: anatomia@amb.edu.pl ical for apoptosis in mitochondria, which are followed with activation of proteolytic cleavage and caspases, such as Bcl-x and Bax. The latter inhibit apoptotic cascade of Bcl-xL, Bcl-2 or Mcl-1 [3,4]. Bcl-2 is a membrane-bound protein that, due to the ability to inactivate free radicals and influence on calcium ions distribution, protects cells from death by apoptosis. Bax protein has a proapoptotic function. It is responsible for causing changes typical for apoptosis in mitochondria, e.g. loss of electrochemical gradient, secretion of cytochrome $\mathrm{c}$ and calcium ions from mitochondrial matrix, which lead to activation of proteolytic processes and caspases. Bax and Bcl-2 protein constitute connections in the form of heterodimers or larger conglomerates. Bcl-xL proteins extend life span of a cell, whereas Bcl-xs has an apoptotic function. Bcl-2 fami- 
Table 1. Expression and intensity of immunohistochemical reaction in B-CLL patients before and after treatment

\begin{tabular}{|c|c|c|c|c|c|c|}
\hline \multirow{3}{*}{$\begin{array}{l}\text { No. of } \\
\text { patients }\end{array}$} & \multicolumn{6}{|c|}{$\mathrm{Bcl}_{2}$} \\
\hline & \multicolumn{3}{|c|}{ before } & \multicolumn{3}{|c|}{ after } \\
\hline & Intensity of reaction & Mean & $\mathrm{SD}$ & Intensity of reaction & Mean & $\mathrm{SD}$ \\
\hline 8 & +++ & 83,6 & 7,4 & +++ & 78,0 & 12,1 \\
\hline 4 & +++ & 85,3 & 3,1 & ++ & 85,7 & 13,5 \\
\hline 1 & ++ & 68,0 & 0 & +++ & 99,0 & 0 \\
\hline 2 & ++ & 62,5 & 14,9 & ++ & 33,0 & 18,4 \\
\hline 1 & + & 42,0 & 0 & +++ & 86,0 & 0 \\
\hline \multirow[t]{2}{*}{2} & + & 66,5 & 6,4 & ++ & 49,5 & 4,9 \\
\hline & \multicolumn{6}{|c|}{$\mathrm{Bax}$} \\
\hline 7 & +++ & 63,6 & 16,6 & +++ & 62,8 & 12,7 \\
\hline 3 & +++ & 62,0 & 9,2 & ++ & 14,7 & 3,5 \\
\hline 1 & +++ & 82,0 & 0 & + & 10,0 & 0 \\
\hline 5 & ++ & 39,0 & 13,9 & +++ & 58,0 & 14,7 \\
\hline 2 & ++ & 53,0 & 1,4 & ++ & 42,0 & 28,3 \\
\hline \multirow[t]{2}{*}{1} & + & 42,0 & 0 & +++ & 86,0 & 0 \\
\hline & \multicolumn{6}{|c|}{$\mathrm{Bcl}_{\mathrm{x}}$} \\
\hline 10 & +++ & 50,2 & 23,8 & +++ & 35,7 & 12,5 \\
\hline 3 & +++ & 47,0 & 16,1 & ++ & 25,3 & 23,4 \\
\hline 2 & ++ & 20,0 & 2,8 & +++ & 38,0 & 0 \\
\hline 2 & ++ & 46,7 & 13,6 & ++ & 22,0 & 3,5 \\
\hline l & + & 62,0 & 0 & +++ & 12,0 & 0 \\
\hline
\end{tabular}

ly proteins regulate life span of cells and therefore play an important role in etiology and survivability of neoplastic cells and acquired resistance to antineoplastic treatment $[4,5]$. Excessive expression of the Bcl-2 protein is associated with a block in apoptosis. Mutations of p53 are associated with drug resistance [6]. New purine nucleoside analogues, such as deoxycoformycin (pentostatin, dCF), 2-chlordeoxyadenosine (2-CdA, cladribine) and fludarabine (FAMP), influence cells in a state of quiescence by inducing apoptosis [7-9]. Histological examination of bone marrow obtained by trephine biopsy (TB) is not obligatory in diagnosis of B-CLL. However, it is a useful diagnostic procedure in monitoring the course of the disease, its prognosis and assessment of MRD (minimal residual disease), i.a. nodular partial remission (nPR) in bone marrow [10-12]. In B-CLL leukaemic cells may infiltrate bone marrow in a diffuse, nodular, interstitial and mixed (nodular/interstitial) pattern. It was observed that the patients with diffuse pattern of infiltration in bone marrow tend to develop more advanced form of the disease and therefore relatively poor prognosis [13].
The aim of the study was to assess expression of $\mathrm{Bcl}-2$, Bax and Bcl-x in neoplastic infiltration in bone marrow in patients with B-CLL treated with nucleoside analogues.

\section{Material and methods}

Bone marrow samples. The bone marrow was obtained by routine $\mathrm{TB}$ of posterior superior iliac spine in accordance with clinical indications from 18 newly diagnosed, untreated patients ( 8 women and 10 men, aged 35-72), with B-CLL at the II, III or IV clinical stage of the disease according to Rai staging system. The diagnosis of B-CLL was strictly confirmed by relevant clinical and cytological findings. TB was performed in every patient as initial examination prior to chemotherapy and after six courses of treatment. Bone marrow tissue material was fixed in so-called "Oxford solution" (100 $\mathrm{ml}$ of $40 \%$ formaldehyde solution, $900 \mathrm{ml}$ distilled water, $8.7 \mathrm{~g}$ Natrium Chloride, $20 \mathrm{ml}$ glacial acetic acid). After fixing (48-72 hours), the cylinder-shaped bone marrow specimens 2 $\mathrm{cm}$ long and $1 \mathrm{~mm}$ in diameter were put into paraffin bars. The bars were cut in sections of $5 \mu \mathrm{m}$ thickness and afterwards underwent routine histological and immunohistochemical procedure. The stage and the pattern of neoplastic infiltration were assessed (diffuse, nodular, interstitial and mixed). 

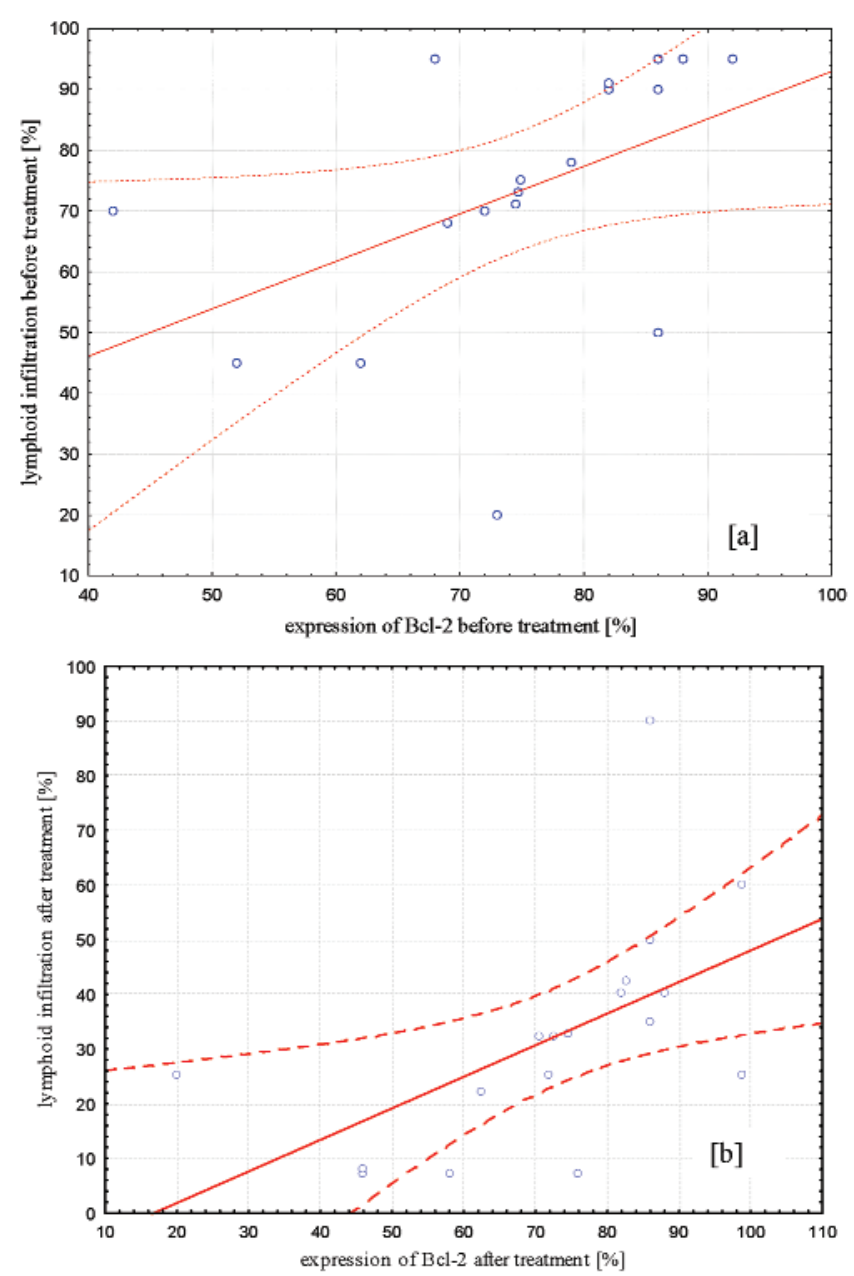

Fig. 1. Correlation between the Bcl-2 expression and the stage of lymphoid infiltration before and after treatment in B-CLL patients.

Immunohistochemistry. The origin of neoplastic cells forming infiltration was confirmed by coupling with anti CD20 and anti CD3 monoclonal antibody (DAKO Cytomation). Immunohistochemical examinations were conducted with the help of specific monoclonal Bcl-2, Bax antibodies and polyclonal Bcl-x antibodies by DAKO Cytomation. Primary antibodies were used by dilution according to the manufacturer's instructions. All sections required microwave heating for receptor retrieval. A detecting kit for Bcl-2 was LSAB+KIT (DAKO Cytomation), and CSA System (DAKO Cytomation) for Bax and Bcl-x. DAB (3, 3'- diaminobenzidine) was used as a chromogen. If the reaction was positive browncoloured antigen-antibody complexes appeared in the position of a protein receptor. Intensity of the positive reaction was defined according to a three grade scale (from + to +++ ): $(+)$ weak staining, $(++)$ moderate staining, $(+++)$ strong staining. Presence of expression of proteins was defined as percentage value of neoplastic cells with positive reaction to anti-Bcl-2, anti-Bax and anti-Bcl$\mathrm{x}$ in regard to total number of cells constituting neoplastic infiltration. Percentage value of neoplastic cells with positive reaction was analysed with regard to morphometric analyser set Soft Imaging System (OLYMPUS). The Bcl-2/Bax ratio before and after treatment was accounted. For negative control the sections underwent the above procedure without primary antibodies. Positive control for Bcl-2, Bax and Bcl-x antibodies was performed using lymph node slices. Control group of the Bcl-2, Bax and Bcl-x physiologic staining constituted five trephine biopsy specimens obtained from patients without proliferative disease.

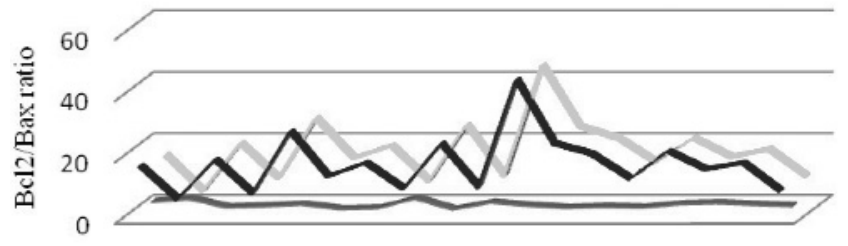

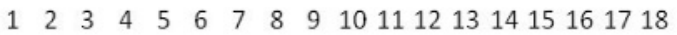

patients

before $\square$ after $\square$ difference

Fig. 2. Bcl-2/Bax ratio before and after treatment in each B-CLL patient.

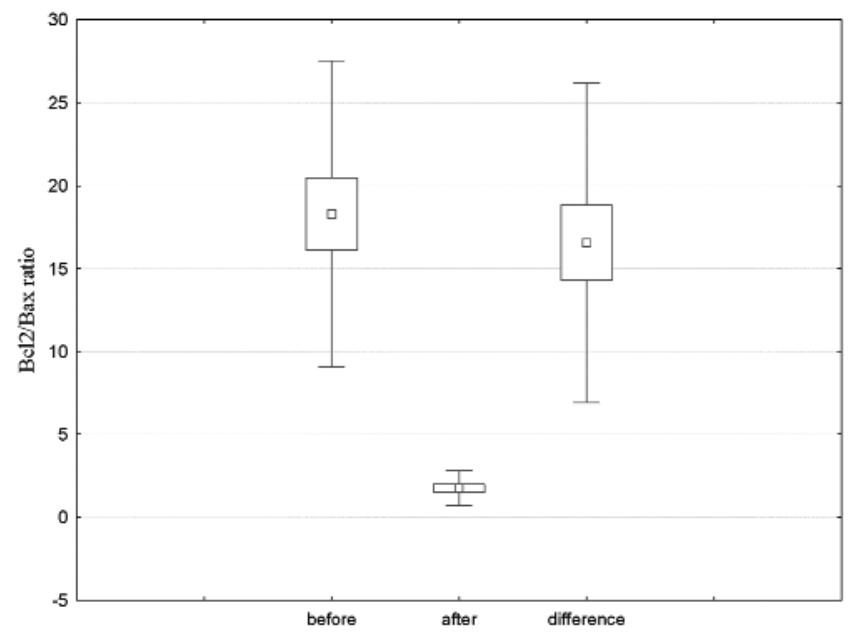

Fig. 3. The mean difference between Bcl-2/Bax ratio in lymphoid infiltration before and after treatment in B-CLL patients.

Ethical issues. The study was approved by local Bioethical Committee of the Medical University of Bialystok no R-I003/204/2005.

Statistical analysis. Obtained results were analysed with the help of the Wilcoxon matched pairs test and Spearman rank-order correlation using the computer program Statistica ${ }^{\mathrm{TM}}$ PL.

\section{Results}

Infiltration of bone marrow by lymphoid cells was observed in all patients before treatment. In fifteen patients bone marrow was infiltrated in a diffuse pattern and in three patients in a nodular pattern. After the treatment the diffuse infiltration persisted only in two patients. In the cases of a nodular pattern before treatment, the stage of infiltration by lymphoid cells after treatment was lower. Statistically significant differences were observed between infiltration stage before treatment and after its completion $(p<0.002)$. Very high percentage value of cells with positive $\mathrm{Bcl}-2$ reaction was observed from $42.0 \%$ in one patient to $85.33 \pm 3.06 \%$ in four patients. After the treatment high 

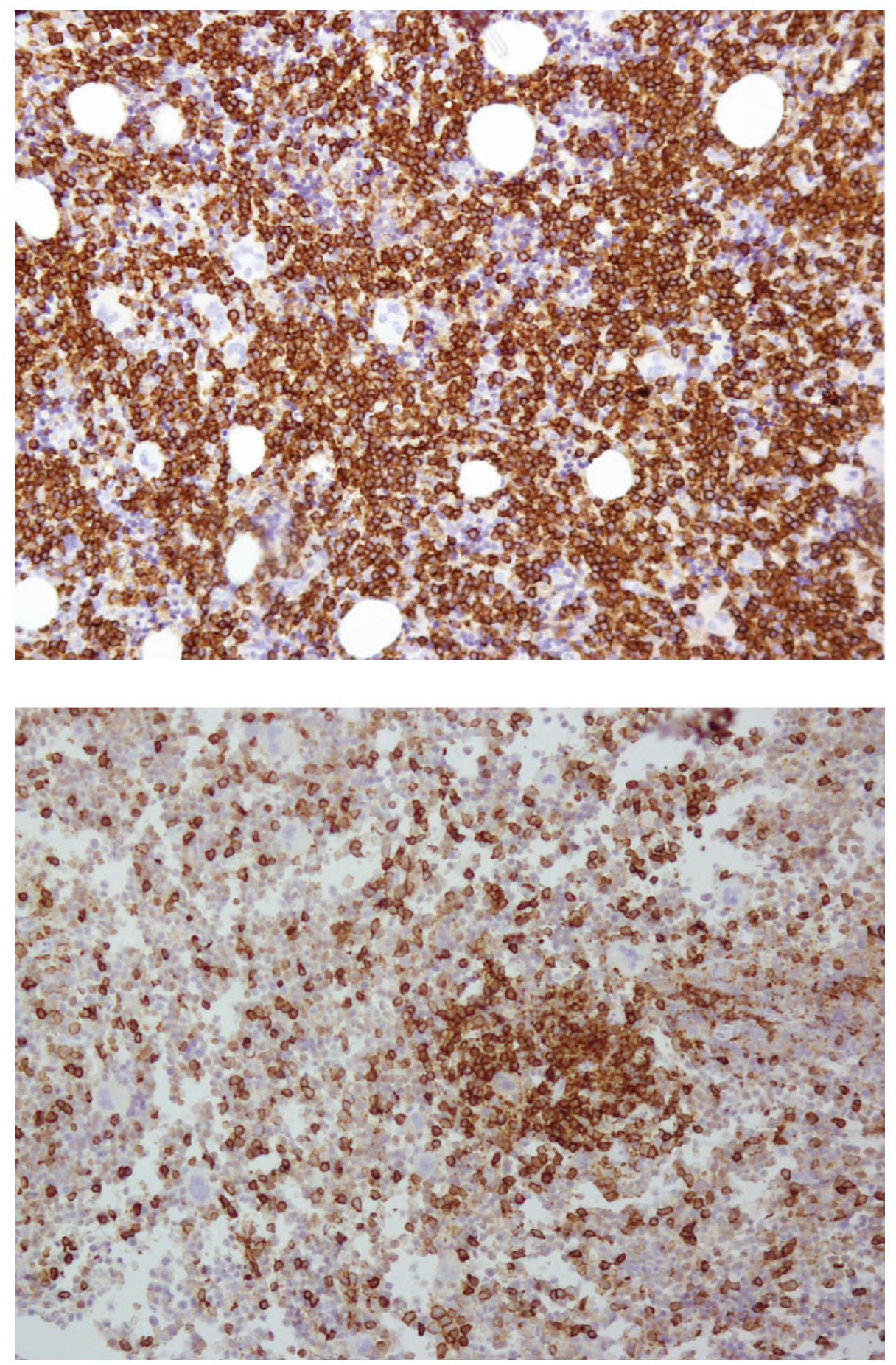

Fig. 4. Bone marrow trephine biopsy section, B-CLL, diffuse infiltration. Lymphoid cells with positive strong staining for Bcl-2 antibody before treatment (magnification $\times 240$ ).
Fig. 5. Bone marrow trephine biopsy section, B-CLL, nodular infiltration. Lymphoid cells with positive strong staining for Bcl-2 antibody after treatment (magnification $\times 240$ ). expression of Bcl-2 was still present in other infiltration cells from $33.0 \pm 18.38 \%$ in two patients to $99.0 \%$ in one patient. The results are presented in Table 1 . Correlation between Bcl-2 expression and the stage of lymphoid infiltration before and after treatment was shown (Fig. 1). Bax expression in infiltration cells was accounted for $39.0 \pm 13.9 \%$ in five patients to $82.0 \%$ in one patient. After the treatment percentage value of the cells with positive anti-Bax reaction constituted from $10.0 \%$ in one patient to $62.8 \pm 12.68 \%$ in seven patients
(Table 1). Bcl-x expression in infiltration cells before treatment was accounted for $36.0 \%$ in one patient to $62.0 \%$ in one patient. After the treatment percentage value of the infiltration cells with positive anti-Bcl-x reaction was accounted for $12.0 \%$ in one patient to $38.0 \%$ in two patients (Table 1 ).

No correlation between Bcl-x and Bax, and the stage of infiltration was found. The high Bcl-2/Bax ratio before treatment was observed (Fig. 2). There was a difference between $\mathrm{Bcl}-2 / \mathrm{Bax}$ ratio before and 
after treatment (Fig. 3). In twelve patients strong staining $(+++)$ of immunohistochemical reaction of infiltration cells with Bcl-2 was observed before treatment (Fig. 4). Strong staining (+++) of immunohistochemical reaction of infiltration cells with anti-Bax appeared in eleven patients. After treatment infiltration cells presented strong staining $(+++)$ of immunohistochemical reaction with anti Bcl-2 in ten patients, whereas with Bax in thirteen patients (Fig. 5). In thirteen patients strong staining $(+++)$ of immunohistochemical reaction of infiltration cells with anti Bcl-x before and after the treatment was observed. In other cases the intensity of the positive reaction was moderate. In single cases the intensity of the positive reaction was weak. Differences between the intensity stage of immunohistochemical reaction of infiltration cells with Bcl-2, Bax and Bcl-x before and after the treatment that appeared were not statistically significant.

\section{Discussion}

Bcl-2 family proteins, by regulating life span of the cells, play important role in etiology and progression of neoplasms. Bcl-2 protein plays a key role in the control of apoptosis [2]. In our study there was high percentage of cells of neoplastic infiltration with positive anti-Bcl-2 reaction before treatment. Correlation between the percentage of lymphoid cells and expression of Bcl-2 protein was shown. This supports observations, that excessive expression of this protein is one of the most important factors in the development of many neoplasms, including CLL. It is also significant in the case of survival of neoplastic cells [6]. Excessive expression of anti-apoptotic Bcl-2 family proteins influences acquired resistance to antineoplastic treatment $[14,16]$. In most cases of our study it turned out that despite the administered treatment and change of the infiltration pattern from diffuse into nodular, the expression of Bcl-2 protein was still observed. This suggests that lymphoid cells with disturbed mechanism of apoptosis and excessive Bcl-2 expression were still present. The nPR is often observed in patients with CLL, which often suggests difficulties in achieving total remission of the disease regardless of administered treatment [11]. Nevertheless, purine nucleoside analogues treatment seems to bear significance. In most cases of our study considerable reduction of the mass of neoplastic cells was observed. These medicines can induce apoptosis in neoplastic cells [7]. Bcl2 is considered as a general apoptosis inhibitor so it is very important to study new drugs like antisense oligonucleotides, which with the help of other drugs would overcome neoplastic cells resistance leading to apoptosis [14-16].

In the literature it has been suggested that in acute myeloid leukaemia, where overexpression of $\mathrm{Bcl}-2$ occurs, the Bcl-2/Bax ratio is more significant than absolute values of these proteins. The higher the ratio is, the worse prognosis of achieving total remission of the disease and event-free survival against recurrence of the disease are [14]. In case of increased values of $\mathrm{Bcl}-2 / \mathrm{Bax}$ ratio the raised cells' resistance to conventional treatment is observed [17]. Our studies revealed correlation between $\mathrm{Bcl}-2$ and $\mathrm{Bax}$ proteins before treatment. The $\mathrm{Bcl}-2 / \mathrm{Bax}$ ratio before treatment in the most of cases was high which can prove worse prognosis and lesser ability to obtain total remission in those patients. Bcl-2 and Bax correlation was not confirmed after treatment.

It has been suggested that neoplastic cells seem to have disturbed mechanism of apoptosis due to Bcl-xL protein $[2,3]$. In our studies polyclonal antibody $\mathrm{Bcl}-\mathrm{x}$ was used. Correlation between Bcl-x and the stage of infiltration by lymphoid cells was not shown before and after treatment. Strong staining of immunohistochemical reaction defined as +++ was observed, which indirectly suggests involvement of this protein in disturbance of apoptosis.

$\mathrm{Bcl}-2$ protein which is present in neoplastic cells is a sensitive marker of MRD in bone marrow [18]. In our studies intensity of immunohistochemical reaction was strong $(+++)$ or moderate $(++)$, which supports the possibility of performing tests on expression of Bcl-2 family proteins in trephine biopsy specimens of bone marrow. Bone marrow examination through TB with the help of immunohistochemical methods and image analysis supports its usefulness in prognosis of the course of the disease, assessment of efficacy of the administered treatment and MRD detection $[11,12,18]$.

\section{References}

[1] Granziero L, Ghia P, Circosta P et al. Survivin in expressed on CD40 stimulation and interfaces proliferation and apoptosis in B-cell chronic leukemia. Blood. 2001;97(9):2777-2783.

[2] Sanchez-Beato M.,Aguilera AS, Piris M.A. Cell cycle deregulation in B-cell lymphomas. Blood. 2003;101(4):1220-1235.

[3] Zhang Y, Dawson M, Mohammad R et al. Induction of apoptosis of human B-CLL and ALL cells by a novel retinoid and its nonretinoidal analog. Blood. 2002;100(8):2917- 2925.

[4] Antonsson B, Martinou JC. Minireview. The Bcl-2 protein family. Exp Cell Res. 2000; 256:50-57.

[ 5] Hermann M, Scholman HJ, Marafioti T, Stein H, Schriever F. Differential expression of apoptosis, bcl-x and c-Myc in normal and malignant lymphoid tissues. Eur J Haematol. 1997; 59:20-30.

[6] Newcomb EW. P53 gene mutations in lymphoid diseases and their possible relevance to drug resistance. Leuk Lymphoma. 1995; 17:211-221.

[ 7] Robak T. The role of nucleoside analogues in the treatment of chronic lymphocytic leukemia- lessons learned from prospective randomized trials. Leuk Lymphoma. 2002;43(3):537-548.

[8] Schriever F, Huhn D. New directions in the diagnosis and treatment of chronic lymphocytic leukaemia. Drugs. 2003;63:953-969. 
[9] Montserrat E, Rozman C. Chronic lymphocytic leukemia: present status. Ann Oncol. 1995;6:219-235.

[10] Cheson BD, Bennett JM, Grever M et al. National Cancer Institute- sponsored working group guidelines for chronic lymphocytic leukemia: revised guidelines for diagnosis and treatment. Blood. 1996;87:4990-4997.

[11] Oudat R, Keating MJ, Lerner S, O'Brien S, Albitar M. Significance of the levels of bone marrow lymphoid infiltrate in chronic lymphocytic leukemia patients with nodular partial remission. Leukemia. 2002;16:632-635.

[12] Sah SP, Matutes E, Wotherspoon AC, Morilla R, Catovsky D A comparison of flow cytometry, bone marrow biopsy, and bone marrow aspirates in the detection of lymphoid infiltration in B cell disorders. J Clin Pathol. 2003;56:129-132.

[13] Bain BJ, Clark DM, Lampert IA, Wilkins BS Lymphoproliferative disorders. In: Bone marrow pathology. 3rd ed. Blackwell Science; 2001:231-331.

[14] Schimmer A, Hedley DW, Penn LZ, Minden MD. Receptorand mitochondrial-mediated apoptosis in acute leukemia: a translational view. Blood. 2001;98(13):3541-3553.
[15] Sorenson CM. Bcl-2 family members and disease. Biochim Biophys Acta. 2004;1644:169-177.

[16] Lowe SW, Lin AW. Apoptosis in cancer. Carcinogenesis. 2000;21(3):485-495.

[17] Pepper C, Hoy T, Bentley P. Elevated Bcl-2/Bax are a consistent feature of apoptosis in B-cell chronic lymphocytic leukaemia and are correlated with in vivo chemoresistance. Leuk Lymphoma. 1998;28:355-361

[18] Gala JL, Guiot Y, Delannoy A, Scheiff JM, Philippe M, Martiat P. Use of image analysis and immunostaining of bone marrow trephine biopsy specimens to quantify residual disease in patients with B- cell chronic lymphocytic leukemia. Mod Pathol. 1999;12:391-399.

Submitted: 22 November, 2007 Accepted after reviews: 23 March, 2008 